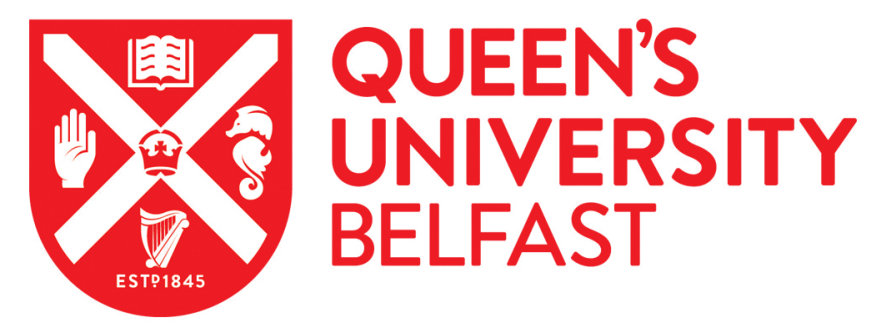

\title{
No Association Between Vitamin D Status and Risk of Barrett's Esophagus or Esophageal Adenocarcinoma: A Mendelian Randomization Study
}

Dong, J., Gharahkhani, P., Chow, W-H., Gammon, M. D., Liu, G., Caldas, C., Wu, A. H., Ye, W., Onstad, L., Anderson, L., Bernstein, L., \& Pharoah, P. (2019). No Association Between Vitamin D Status and Risk of Barrett's Esophagus or Esophageal Adenocarcinoma: A Mendelian Randomization Study. Clinical gastroenterology and hepatology : the official clinical practice journal of the American Gastroenterological Association. https://doi.org/10.1016/j.cgh.2019.01.041

Published in:

Clinical gastroenterology and hepatology : the official clinical practice journal of the American

Gastroenterological Association

Document Version:

Peer reviewed version

Queen's University Belfast - Research Portal:

Link to publication record in Queen's University Belfast Research Portal

\section{Publisher rights}

Copyright 2019 Elsevier.

This manuscript is distributed under a Creative Commons Attribution-NonCommercial-NoDerivs License

(https://creativecommons.org/licenses/by-nc-nd/4.0/), which permits distribution and reproduction for non-commercial purposes, provided the author and source are cited.

\section{General rights}

Copyright for the publications made accessible via the Queen's University Belfast Research Portal is retained by the author(s) and / or other copyright owners and it is a condition of accessing these publications that users recognise and abide by the legal requirements associated with these rights.

\section{Take down policy}

The Research Portal is Queen's institutional repository that provides access to Queen's research output. Every effort has been made to ensure that content in the Research Portal does not infringe any person's rights, or applicable UK laws. If you discover content in the Research Portal that you believe breaches copyright or violates any law, please contact openaccess@qub.ac.uk. 


\section{Vitamin D status and the risks of Barrett's esophagus and esophageal adenocarcinoma: a Mendelian randomization study}

Short title: Vitamin D and the risks of Barrett's esophagus and esophageal adenocarcinoma Jing Dong, ${ }^{1}$ Puya Gharahkhani, ${ }^{2}$ Wong-Ho Chow, ${ }^{3}$ Marilie D. Gammon, ${ }^{4}$ Geoffrey Liu, ${ }^{5}$ Carlos Caldas, ${ }^{6}$ Anna H. Wu, ${ }^{7}$ Weimin Ye, ${ }^{8}$ Lynn Onstad, ${ }^{9}$ Lesley A. Anderson, ${ }^{10}$ Leslie Bernstein, ${ }^{11}$ Paul D. Pharoah, $, 2,13$ Harvey A. Risch, ${ }^{14}$ Douglas A. Corley, ${ }^{15,16}$ Rebecca C. Fitzgerald, ${ }^{17}$ Stomach and Esophageal Cancer Study (SOCS) Consortium, Prasad G. Iyer, ${ }^{18}$ Brian J. Reid, ${ }^{9}$ Jesper Lagergren, ${ }^{19,20}$ Nicholas J. Shaheen, ${ }^{21}$ Thomas L. Vaughan, ${ }^{9}$ Stuart MacGregor, ${ }^{2}$ Sharon Love, ${ }^{22}$, Claire Palles, ${ }^{23}$ Ian Tomlinson, ${ }^{23}$ Ines Gockel, ${ }^{24}$ Andrea May, ${ }^{25}$ Christian Gerges, ${ }^{26}$ Mario Anders, ${ }^{27,28}$ Anne C. Böhmer, ${ }^{29,30}$ Jessica Becker, $, 31,32$ Nicole Kreuser ${ }^{24}$ Rene Thieme,${ }^{24}$ Tania Noder,${ }^{27}$ Marino Venerito, ${ }^{31}$ Lothar Veits, ${ }^{32}$ Thomas Schmidt,${ }^{33}$ Claudia Schmidt, ${ }^{34}$ Jakob R. Izbicki, ${ }^{35}$ Arnulf H. Hölscher,,${ }^{34}$ Hauke Lang, ${ }^{36}$ Dietmar Lorenz, ${ }^{37}$ Brigitte Schumacher, ${ }^{38}$ Rupert Mayershofer, ${ }^{39}$ Yogesh Vashist,,${ }^{35,40}$ Katja Ott, ${ }^{33,41}$ Michael Vieth, ${ }^{32}$ Josef Weismüller, ${ }^{42}$ Markus M. Nöthen, ${ }^{29,30}$ Susanne Moebus, ${ }^{43}$ Michael Knapp, ${ }^{44}$ Wilbert H. M. Peters,${ }^{45}$ Horst Neuhaus, ${ }^{29}$ Thomas Rösch, ${ }^{26}$ Christian Ell, ${ }^{25}$ Janusz Jankowski, ${ }^{46}$ Johannes Schumacher, ${ }^{29,30}$ Rachel E. Neale, ${ }^{47}$ David C. Whiteman, ${ }^{48}$ Aaron P. Thrift*1,49

${ }^{1}$ Dan L Duncan Comprehensive Cancer Center, Baylor College of Medicine, Houston, TX, USA.

${ }^{2}$ Statistical Genetics, QIMR Berghofer Medical Research Institute, Brisbane, QLD, Australia.

${ }^{3}$ Department of Epidemiology, MD Anderson Cancer Center, Houston, TX, USA.

${ }^{4}$ Department of Epidemiology, University of North Carolina, Chapel Hill, NC, USA.

${ }^{5}$ Pharmacogenomic Epidemiology, Ontario Cancer Institute, Toronto, Ontario, Canada M5G 2M9.

${ }^{6}$ Cancer Research UK, Cambridge Institute, Cambridge, UK. 
${ }^{7}$ Department of Preventive Medicine, University of Southern California/Norris Comprehensive Cancer Center, Los Angeles, CA, USA.

${ }^{8}$ Department of Medical Epidemiology and Biostatistics, Karolinska Institutet, Stockholm, Sweden. ${ }^{9}$ Division of Public Health Sciences, Fred Hutchinson Cancer Research Center, Seattle, WA, USA. ${ }^{10}$ Centre for Public Health, Queen’s University Belfast, Belfast, Northern Ireland.

${ }^{11}$ Department of Population Sciences, Beckman Research Institute and City of Hope Comprehensive Cancer Center, Duarte, CA, USA.

${ }^{12}$ Department of Oncology, University of Cambridge, Cambridge, UK.

${ }^{13}$ Department of Public Health and Primary Care, University of Cambridge, Cambridge, UK.

${ }^{14}$ Department of Chronic Disease Epidemiology, Yale School of Public Health, New Haven, CT, USA.

${ }^{15}$ Division of Research, Kaiser Permanente Northern California, Oakland, CA, USA.

${ }^{16}$ San Francisco Medical Center, Kaiser Permanente Northern California, San Francisco, CA, USA.

${ }^{17}$ Medical Research Council (MRC) Cancer Unit, Hutchison-MRC Research Centre, University of Cambridge, Cambridge, UK.

${ }^{18}$ Division of Gastroenterology and Hepatology, Mayo Clinic, Rochester, MN, USA.

${ }^{19}$ Department of Molecular Medicine and Surgery, Karolinska Institutet, Stockholm, Sweden.

${ }^{20}$ School of Cancer and Pharmaceutical Sciences, King's College London, London, UK.

${ }^{21}$ Division of Gastroenterology and Hepatology, University of North Carolina School of Medicine, Chapel Hill, NC, USA.

${ }^{22}$ Centre for Statistics in Medicine and Oxford Clinical Trials Research Unit, Oxford, UK

${ }^{23}$ Institute of Cancer and Genomic Sciences, University of Birmingham, Birmingham, UK

${ }^{24}$ Department of Visceral, Transplant, Thoracic and Vascular Surgery, University Hospital of Leipzig, Leipzig, Germany

${ }^{25}$ Department of Medicine II, Sana Klinikum, Offenbach, Germany

${ }^{26}$ Department of Internal Medicine II, Evangelisches Krankenhaus, Düsseldorf, Germany 
${ }^{27}$ Department of Interdisciplinary Endoscopy, University Hospital Hamburg-Eppendorf, Hamburg, Germany

${ }^{28}$ Department of Gastroenterology and Interdisciplinary Endoscopy, Vivantes Wenckebach-Klinikum, Berlin, Germany

${ }^{29}$ Institute of Human Genetics, University of Bonn, Bonn, Germany

${ }^{30}$ Department of Genomics, Life \& Brain Center, University of Bonn, Bonn, Germany

${ }^{31}$ Department of Gastroenterology, Hepatology and Infectious Diseases, Otto-von-Guericke University Hospital, Magdeburg, Germany

${ }^{32}$ Institute of Pathology, Klinikum Bayreuth, Bayreuth, Germany

${ }^{33}$ Department of General, Visceral and Transplantation Surgery, University of Heidelberg, Heidelberg, Germany

${ }^{34}$ Department of General, Visceral and Cancer Surgery, University of Cologne, Cologne, Germany

${ }^{35}$ Department of General, Visceral and Thoracic Surgery, University Medical Center Hamburg-Eppendorf, University of Hamburg, Hamburg, Germany

${ }^{36}$ Department of General, Visceral and Transplant Surgery, University Medical Center, University of Mainz, Mainz, Germany

${ }^{37}$ Department of General, Visceral and Thoracic Surgery, Klinikum Darmstadt, Darmstadt, Germany

${ }^{38}$ Department of Internal Medicine and Gastroenterology, Elisabeth Hospital, Essen, Germany

${ }^{39}$ Gastroenterologie am Burgweiher, Bonn, Germany

${ }^{40}$ Kantonsspital Aarau, Aarau, Switzerland

${ }^{41}$ Department of General, Visceral and Thorax Surgery, RoMed Klinikum Rosenheim, Rosenheim, Germany

${ }^{42}$ Gastroenterologische Gemeinschaftspraxis, Koblenz, Germany

${ }^{43}$ Centre of Urban Epidemiology, Institute of Medical Informatics, Biometry and Epidemiology, University of Essen, Essen, Germany 
${ }^{44}$ Institute for Medical Biometry, Informatics, and Epidemiology, University of Bonn, Bonn, Germany

${ }^{45}$ Department of Gastroenterology, Radboud University Nijmegen Medical Center, Nijmegen, The Netherlands

${ }^{46}$ Royal College of Surgeons in Ireland, Dublin, Ireland

${ }^{47}$ Cancer Aetiology and Prevention, QIMR Berghofer Medical Research Institute, Brisbane, QLD, Australia

${ }^{48}$ Cancer Control, QIMR Berghofer Medical Research Institute, Brisbane, QLD, Australia.

${ }^{49}$ Section of Epidemiology and Population Sciences, Department of Medicine, Baylor College of Medicine, Houston, TX, USA.

Grant support: This work was primarily funded by National Institutes of Health (R01CA136725). The funders of the study had no role in the design, analysis, or interpretation of the data, nor in writing or publication decisions related to this article. JD was supported by a Research Training Grant from the Cancer Prevention and Research Institute of Texas (CPRIT; RP160097). PG was supported by a grant from National Health and Medical Research Council of Australia (1123248). GL was supported by the Alan B. Brown Chair in Molecular Genomics and by the CCO Chair in Experimental Therapeutics and Population Studies. The University of Cambridge received salary support for PDP from the NHS in the East of England through the Clinical Academic Reserve. BJR was supported by a grant (P01CA91955) from the National Institutes of Health /National Cancer Institute. NJS was supported by a grant (P30 DK034987) from the National Institutes of Health. TLV was supported by National Institutes of Health Established Investigator Award K05CA124911. SM was supported by an Australian Research Council Future Fellowship. DCW and REN were supported by Research Fellowships from the National Health and Medical Research Council of Australia.

Conflicts of Interest: The authors disclose no potential conflicts of interest. 
Correspondence to: Aaron P. Thrift, Ph.D., Dan L Duncan Comprehensive Cancer Center, Baylor College of Medicine, One Baylor Plaza, MS: BCM305, Houston, Texas 77030-3498. Tel: 713-798-9107; Fax: 713-798-3658; E-mail: aaron.thrift@bcm.edu

Specific author contributions: APT had full access to all of the data in the study and takes responsibility for the integrity of the data and the accuracy of the data analysis. Study concept and design: JD, DCW, APT. Obtained the data: WHC, MDG, GL, AHW, WY, LAA, LB, PDP, HAR, DAC, RCF, PGI, BJR, JL, NJS, TLV, JJ, JS, DCW. Statistical analysis and interpretation of data: JD, PG, DCW, APT. Drafting of the manuscript: JD, PG, DCW, APT. Critical review of the manuscript for important intellectual content: JD, PG, WHC, MDG, GL, CC, AW, WY, LO, LAA, LB, PDP, HAR, DAC, RCF, PGI, BJR, JL, NJS, TLV, SMG, SL, CP, IT, IG, AM, CG, MA, ACB, JB, NK, RT, TN, MV, LV, TS, CS, JRI, AHH, HL, DL, BS, RM, YV, KO, MV, JW, MMN, SM, MK, WMP, HN, TR, CE, JJ, SJ, REN, DCW, APT. Study supervision: APT. All authors read and approved the final version for submission.

Abbreviations: esophageal adenocarcinoma, EAC; Barrett's esophagus, BE; 25 hydroxy vitamin D, 25(OH)D. 


\begin{abstract}
Background Epidemiological studies on circulating concentrations of 25 hydroxy vitamin D (25(OH)D) and risks of esophageal adenocarcinoma (EAC) have shown conflicting results. Here we conducted a Mendelian randomization study to determine the associations between circulating concentrations of 25 hydroxy vitamin D (25(OH)D) and risks of EAC and its precursor, Barrett's esophagus (BE).
\end{abstract}

Methods We conducted a Mendelian randomization study using a two-sample (summary data) approach. Six single-nucleotide polymorphisms (SNPs) (rs3755967, rs10741657, rs12785878, rs10745742, rs8018720 and rs17216707) associated with circulating 25(OH)D concentrations were used as instrumental variables. Data from 6,167 BE patients, 4,112 EAC patients and 17,159 control participants were from studies participating in the Barrett's and Esophageal Adenocarcinoma Consortium (BEACON), as well as studies from Bonn, Germany, and Cambridge and Oxford, United Kingdom. Analyses were performed separately for BE and EAC.

Results Overall, we found no evidence for an association between genetically estimated $25(\mathrm{OH}) \mathrm{D}$ concentration and the risk of BE or EAC. The odds ratio (OR) per $20 \mathrm{nmol} / \mathrm{L}$ increase in genetically estimated 25(OH)D concentration for BE risk estimated by combining the individual SNP association using inverse variance weighting was 1.21 (95\% confidence interval, $\mathrm{CI}, 0.77-1.92 ; P=0.41)$. The OR for EAC risk estimated by combining the individual SNP association using inverse variance weighting was $0.68(95 \% \mathrm{CI}, 0.39-1.19 ; P=0.18)$.

Conclusions This Mendelian randomization study found that low genetically estimated 25(OH)D concentrations were not associated with the risks of BE or EAC.

Key Words: Vitamin D, Barrett's esophagus, esophageal adenocarcinoma, Mendelian randomization study 


\section{INTRODUCTION}

The incidence of esophageal adenocarcinoma (EAC) has increased rapidly during the last four decades (1), and despite advances in early detection and treatment, the 5-year survival in EAC remains less than 20\% (2). Barrett's esophagus (BE), metaplastic columnar epithelial overgrowth of the distal esophagus, is the precursor for EAC (3). Established risk factors for EAC and BE include gastro-esophageal reflux disease $(4,5)$, obesity $(6)$, and tobacco smoking $(7,8)$. Epidemiological studies have examined the association between vitamin D and risk of EAC, with conflicting results (9-11). However, these studies have been limited by relatively small sample sizes $(<500$ EAC patients) and by potential biases, including reverse causation that may arise if serum vitamin D levels are measured at or close to the date of cancer diagnosis.

In the absence of convincing evidence from epidemiologic studies, Mendelian randomization is an alternative approach for exploring possible associations between $25(\mathrm{OH}) \mathrm{D}$ concentration and risks of $\mathrm{BE}$ and EAC (12). This approach uses instrumental variables (e.g., genetic variants as proxy markers of directly measured risk factors) to make inferences about the relation between an exposure (e.g., low $25(\mathrm{OH}) \mathrm{D}$ concentration) and outcome (e.g., $\mathrm{BE}$ and $\mathrm{EAC})(12-14)$. If $25(\mathrm{OH}) \mathrm{D}$ is related to risk of $\mathrm{BE}$ or EAC, then the variability in $25(\mathrm{OH}) \mathrm{D}$ that is due to genotype should confer differences in BE or EAC risk directly attributable to $25(\mathrm{OH}) \mathrm{D}$. Since alleles are randomly allocated from parents to offspring at conception, the association between genotype and outcome is not confounded by other factors. Furthermore, genetic variants are measured reliably and are not affected by disease status, thus avoiding recall bias. However, a strong instrumental variable and large sample size are required for an unbiased Mendelian randomization study. The availability of large collections of genome-wide data on phenotypes, such as $25(\mathrm{OH}) \mathrm{D}$ concentration, and disease traits within international consortia represents an opportunity to use this approach. Here, we report the findings of a Mendelian randomization study examining associations between genetically estimated circulating $25(\mathrm{OH}) \mathrm{D}$ concentration and risks of BE and EAC. The selected six $25(\mathrm{OH}) \mathrm{D}$-associated SNPs explained $\sim 3 \%$ of the variance of $25(\mathrm{OH}) \mathrm{D}$ concentration. 


\section{METHODS}

In the absence of a dataset with complete individual-level genetic data, $25(\mathrm{OH}) \mathrm{D}$ concentration levels, and BE/EAC status we applied a standard two-sample approach that uses GWAS summary-level data to assess indirect associations (15). That is, we first estimated the single nucleotide polymorphism (SNP)disease associations from one study sample and the SNP-exposure associations in another study sample. Second, we used the Wald-type ratio estimator to calculate the Mendelian randomization estimate for the association between genetically estimated $25(\mathrm{OH}) \mathrm{D}$ concentration and risks of BE and EAC.

\section{SNP-25(OH)D associations}

A study involving 79,366 participants of European descent from 31 epidemiological studies conducted in Europe and North America, identified six SNPs associated with 25(OH)D levels at genome-wide significance (i.e., $\left.P<5 \times 10^{-8}\right)(16)$. These six SNPs are uncorrelated and are located in or close to the following genes: $G C$ (rs3755967), CYP2RI (rs10741657), DHCR7 (rs12785878), AMDHD1 (rs 10745742), SEC23A (rs8018720) and CYP24A1 (rs17216707). These genes have been shown to encode enzymes and carrier proteins involved in vitamin D synthesis or metabolism (16). As serum 25(OH)D concentration levels were not measured in cases and controls included in our BE/EAC studies, we instead retrieved summary-level data for the associations between these six SNPs and 25(OH)D directly from the published paper (16). In brief, that study estimated the associations between each individual SNP and natural log-transformed 25(OH)D concentration using linear regression models adjusted for month of sample collection, age, sex, body mass index, principal components of ancestry, and laboratory batch, where relevant. The analyses were conducted separately for each cohort, and the results were summarized using fixed-effects inverse variance weighted meta-analysis (16). The estimated magnitudes of association (i.e., $\beta$ coefficients) for the six SNP-25(OH)D associations and their standard errors are shown in Table 1. 


\section{SNP-BE and SNP-EAC associations}

We obtained individual-level epidemiologic and genetic data from 6,167 BE patients, 4,112 EAC patients, and 17,159 control participants from 15 epidemiologic studies from North America, Europe, and Australia included in the Barrett's and Esophageal Adenocarcinoma Consortium (BEACON; http://beacon.tlvnet.net/), as well as from studies from Bonn, Germany; Cambridge, United Kingdom; and Oxford, United Kingdom (17-20). The numbers of participants in each study are shown in Table 2. We have reported detailed information on study participants, genotyping, and imputation previously (17). Briefly, all participants were of European ancestry, and DNA samples extracted from blood or saliva were genotyped on high-density SNP arrays (Illumina, San Diego, CA, USA). BE patients were identified by histopathological diagnosis of intestinal metaplasia, and patients with EAC had a histopathologically confirmed diagnosis of adenocarcinoma. Each contributing study was performed under institutional review board approval and all participants gave informed consent. Standard quality control and imputation were performed for each study.

We used a two-stage analytic approach to estimate SNP-BE and SNP-EAC associations. Analyses were performed separately for each of the six SNPs, as well as separately for BE and EAC. First, study-specific (i.e., for BEACON, Bonn, Cambridge, and Oxford) odds ratios (OR) and corresponding 95\% confidence intervals $(95 \% \mathrm{CI})$ were estimated fitting unconditional logistic regression models which adjusted for age, sex and study-specific top principal components of ancestry, under an additive genetic model using allelic dosage. Second, for each SNP, the study-specific adjusted ORs were pooled to create a summary OR, using a fixed-effects inverse variance-weighting approach.

\section{Mendelian randomization estimate for the association with genetically estimated 25(OH)D}

We used a two-stage analytic approach to estimate the instrumental variable association between genetically estimated $25(\mathrm{OH}) \mathrm{D}$ concentration and risks of BE and EAC. In the first stage, for each 
instrumental variable (i.e., separately for the six SNPs), we used the Wald-type ratio estimator to compute the Mendelian randomization weighted estimates for any influence of genetically estimated $25(\mathrm{OH}) \mathrm{D}$ on risks of BE and EAC (21), and the Delta method to estimate the standard errors (22). In the second stage, the six Mendelian randomization estimates were pooled to create a summary instrumental variable OR, using an inverse-variance weighted meta-analysis approach (23). We scaled estimates to a $20-\mathrm{nmol} / \mathrm{L}$ change in $25(\mathrm{OH}) \mathrm{D}$ concentration, which is approximately the inter-tertile range ( $66^{\text {th }}$ percentile to $33 \mathrm{rd}$ percentile) observed in a large European study.(24) We evaluated the presence and magnitude of heterogeneity across the six Mendelian randomization estimates with the Cochran Q and $I^{2}$ statistics (25). All statistics were two sided and all analyses were conducted using Stata 14.1 (StataCorp, College Station, TX).

\section{Assessment of pleiotropy}

In order to have a valid interpretation for the Mendelian randomization analysis, our instrumental variable (the SNPs) must be independently associated with circulating 25(OH)D concentration and associated with BE or EAC only through its association with circulating $25(\mathrm{OH}) \mathrm{D}$ concentration $(26,27)$. To assess and account for pleiotropy (where a SNP may affect more than one phenotypic characteristic), we employed the MR-Egger regression method (an adaptation of the Egger regression in a meta-analysis) (28). The intercept statistically significantly away from zero is a valid test of directional horizontal pleiotropy, whereas the slope of the MR-Egger regression is the horizontal pleiotropy-adjusted estimated magnitude of association. We used a weighted median method to diagnose and protect against invalid genetic instruments (29), and searched the literature and publicly available GWAS databases for associations between the SNPs and other phenotypes (30).

\section{Power}


Assuming the 6 SNPs above explain $3 \%$ of the variance in $25(\mathrm{OH}) \mathrm{D}$ and with $\alpha=0.05$, our study had $80 \%$ power to detect an OR of 1.25 or higher. 


\section{RESULTS}

\section{SNP-BE and SNP-EAC associations}

The associations between each of the six 25(OH)D-associated SNPs and the risks of BE and EAC are shown in Table 3. Only the SNP rs12785878 was nominally associated with the risk of BE (per copy of the T allele, summary OR, $1.07 ; 95 \% \mathrm{CI}, 1.02-1.13 ; P=0.008)$ and none were associated with the risk of EAC (all $P$ values $>0.1$ ). The magnitude and direction of the individual SNP-BE or SNP-EAC associations were consistent across the 4 datasets, with no evidence of between-study heterogeneity (all $\mathrm{I}^{2}$ $<42 \%)$.

\section{Mendelian randomization estimate for the association with genetically estimated 25(OH)D}

The results of the instrumental variables analyses are shown in Figure 1. They show no associations between genetically estimated 25(OH)D concentration and the risk of BE (per $20 \mathrm{nmol} / \mathrm{L}$ increase in $25(\mathrm{OH}) \mathrm{D}$, summary instrumental variable $\mathrm{OR}, 1.21 ; 95 \% \mathrm{CI}, 0.77-1.92)$. We found little heterogeneity in the estimated magnitudes of association between the six individual SNP variables $\left(\mathrm{I}^{2}=46.8 \%, P_{\text {heterogeneity }}\right.$ $=0.09)$. Likewise, in the instrumental variables analysis of EAC, there were no associations with genetically estimated $25(\mathrm{OH}) \mathrm{D}$ concentration (per $20 \mathrm{nmol} / \mathrm{L}$ increase in $25(\mathrm{OH}) \mathrm{D}$, summary instrumental variable OR, $\left.0.68 ; 95 \% \mathrm{CI}, 0.39-1.19 ; \mathrm{I}^{2}=0 \%, P_{\text {heterogeneity }}=0.99\right)$.

\section{Assumptions of Mendelian randomization}

To satisfy the first assumption that the instrumental variable is associated with the exposure of interest, we selected only SNPs with genome-wide statistically significant association with 25(OH)D concentration. We found no evidence for pleiotropy, with an MR-Egger intercept of $-0.005(P=0.79)$ for BE and $0.007(P=0.75)$ for EAC. The weighted median method also yielded no statistically significant estimates (Supplementary Table 1). Finally, we evaluated whether the SNPs that we used as instrumental variables for 25(OH)D were associated with various other phenotypes. As shown in Supplementary Table 
2, we did not find any associations at genome-wide significance $\left(P<5 \times 10^{-8}\right)$, other than rs 17216707 with eGFR creatinine $\left(P=6 \times 10^{-13}\right)$. 


\section{DISCUSSION}

In this Mendelian randomization study, we used genetic variants as instrumental variables for $25(\mathrm{OH}) \mathrm{D}$ and examined associations between 25(OH)D and risks of BE and EAC. We found no evidence that $25(\mathrm{OH}) \mathrm{D}$ concentration, as predicted by genotype, was associated with risks of BE or EAC. Thus, the study provides little evidence that $25(\mathrm{OH}) \mathrm{D}$ concentration is associated with risk of BE or EAC.

Observational studies have examined the association between 25(OH)D concentration and the risk of various cancers. A large number of case-control studies have reported conflicting results; however, these studies are susceptible to reverse causation. Likewise, results from prospective cohort studies have been inconclusive as a result of measuring $25(\mathrm{OH}) \mathrm{D}$ concentration at relatively unspecified times (anywhere from 0 to $20+$ years) in the past before diagnosis. Given the limitations of these observational studies, it remains difficult to draw causal inferences, especially when inconsistent results are observed. Therefore, several Mendelian randomization studies have been recently conducted to examine associations between genetically predicted 25(OH)D concentration and risk of specific cancers. The advantage of Mendelian randomization studies is that the estimated magnitudes of associations are less likely to be affected by confounding and recall bias. A Mendelian randomization study involving data from 70,563 cancer patients and 84,418 control participants found that $25(\mathrm{OH}) \mathrm{D}$ concentration, as predicted by genotype, was not associated with the risks of prostate, breast, lung, colorectal, ovarian, pancreatic cancers or neuroblastoma (31). Likewise, a Mendelian randomization study found no association between genetically predicted $25(\mathrm{OH}) \mathrm{D}$ concentration and risks of oral or oropharyngeal cancer (32). Another Mendelian randomization study indicated an increased risk of ovarian cancer (OR, 1.27; 95\% CI, 1.061.51) per $20 \mathrm{nmol} / \mathrm{L}$ decrease in genetically predicted $25(\mathrm{OH}) \mathrm{D}$ concentrations in 10,065 ovarian cancer and 21,654 controls (33), but this association was not confirmed in a subsequent Mendelian randomization study of ovarian cancer.(31) 
Few studies, and no Mendelian randomization studies, have examined associations between 25(OH)D and risks of BE and EAC $(9,11,34)$. A nested case-control study from the Cohort Consortium Vitamin D Pooling Project of Rarer Cancers reported no association between circulating 25(OH)D and the risk of EAC $(\mathrm{N}=104)$ (34). A recent meta-analysis investigating associations between makers of vitamin $\mathrm{D}$ exposure (i.e., ultraviolet B exposure, vitamin D intake and vitamin D status) and risk of BE or EAC also found no association (11). Conversely, a case-control study in Australia reported a decreased risk of EAC with higher ultraviolet B exposure although, even if a true finding, this is not necessarily due to vitamin D (9). While there is some evidence that BE patients are often vitamin D insufficient $(35,36)$, studies to date have found no association between vitamin D intake and the risk of BE (10), and vitamin D supplementation has been shown to have no effect on risk of neoplastic progression in BE patients (35). Finally, a BE registry cohort study found no association between circulating $25(\mathrm{OH}) \mathrm{D}$ concentration and risk of prevalent or incident dysplasia or EAC in BE patients (36). The findings from the present Mendelian randomization study provide further evidence that $25(\mathrm{OH}) \mathrm{D}$ concentration is not associated with the risk of BE or EAC. Interestingly, the point estimates (i.e., ORs) for BE and EAC show opposite direction of effect for some of these SNPs, with evidence of moderate heterogeneity in the associations with BE but no heterogeneity for associations with EAC. Nonetheless, the point estimates are not significantly different from zero. Thus, a chance finding may explain the opposite directions.

Strengths of the current study include the high quality of the case-control and cohort studies used. The majority of the studies were population-based in design. Compared to results from traditional analyses of observational studies, the risk estimates using instrumental variables are less likely to be affected by confounding and other types of bias. We used six SNPs known to be associated with 25(OH)D concentration, all located in or near genes encoding enzymes and carrier proteins involved in vitamin D synthesis or metabolism, which provided a strong and valid instrumental variable for $25(\mathrm{OH}) \mathrm{D}$ 
concentration. Furthermore, through various statistical tests and database searches, we found little evidence of pleiotropic effects.

Our study also has limitations. Although we used a large worldwide consortial sample of patients, our study population was relatively small compared to other Mendelian randomization studies of vitamin D. However, few additional BE and EAC patients with non-genetic and genetic data are currently available, so such work may require additional time for study patients to accrue. While our findings are consistent with vitamin D not having a large causal effect on $\mathrm{BE} / \mathrm{EAC}$ risk, as the six $25(\mathrm{OH}) \mathrm{D}$-associated SNPs explain only $\sim 3 \%$ of the variance of $25(\mathrm{OH}) \mathrm{D}$ concentration our study may have nonetheless been underpowered to detect medium to small effects. We are unable to evaluate whether the associations between 25(OH)D concentration and the risks of BE and EAC are nonlinear. It is possible that in populations with low $25(\mathrm{OH}) \mathrm{D}$ due to limited sun exposure, genetically determined variation in $25(\mathrm{OH}) \mathrm{D}$ may be associated with BE or EAC. Finally, we used summary-level data, which did not allow us to stratify analyses by covariates of interest, such as age, sex, smoking, body mass index, and gastroesophageal reflux symptoms.

In conclusion, this Mendelian randomization study provides little evidence that genetically estimated circulating $25(\mathrm{OH}) \mathrm{D}$ concentration is associated with the risk of BE and EAC. 
Figure Legend

Figure 1A. Mendelian randomization estimates of the association between increased 25(OH)D concentration and risk of Barrett's esophagus. The OR estimates are per $20 \mathrm{nmol} / \mathrm{L}$ increase in 25(OH)D concentration. The hollow diamond represents the overall IV estimate with its 95\% CI.

Figure 1B. Mendelian randomization estimates of the association between increased 25(OH)D concentration and risk of esophageal adenocarcinoma. The OR estimates are per $20 \mathrm{nmol} / \mathrm{L}$ increase in $25(\mathrm{OH}) \mathrm{D}$ concentration. The hollow diamond represents the overall IV estimate with its $95 \% \mathrm{CI}$. 


\section{REFERENCES}

1. Thrift AP, Whiteman DC. The incidence of esophageal adenocarcinoma continues to rise: analysis of period and birth cohort effects on recent trends. Ann Oncol. 2012;23(12):3155-62.

2. Thrift AP. The epidemic of oesophageal carcinoma: Where are we now? Cancer Epidemiol. 2016;41:88-95.

3. Reid BJ, Li X, Galipeau PC, Vaughan TL. Barrett's oesophagus and oesophageal adenocarcinoma: time for a new synthesis. Nat Rev Cancer. 2010;10(2):87-101.

4. Cook MB, Corley DA, Murray LJ, Liao LM, Kamangar F, Ye W, et al. Gastroesophageal reflux in relation to adenocarcinomas of the esophagus: a pooled analysis from the Barrett's and Esophageal Adenocarcinoma Consortium (BEACON). PLoS One. 2014;9(7):e103508.

5. Thrift AP, Kramer JR, Qureshi Z, Richardson PA, El-Serag HB. Age at onset of GERD symptoms predicts risk of Barrett's esophagus. Am J Gastroenterol. 2013;108(6):915-22.

6. Thrift AP, Shaheen NJ, Gammon MD, Bernstein L, Reid BJ, Onstad L, et al. Obesity and risk of esophageal adenocarcinoma and Barrett's esophagus: a Mendelian randomization study. Journal of the National Cancer Institute. 2014;106(11).

7. Cook MB, Kamangar F, Whiteman DC, Freedman ND, Gammon MD, Bernstein L, et al. Cigarette smoking and adenocarcinomas of the esophagus and esophagogastric junction: a pooled analysis from the international BEACON consortium. Journal of the National Cancer Institute. 2010;102(17):1344-53.

8. Cook MB, Shaheen NJ, Anderson LA, Giffen C, Chow WH, Vaughan TL, et al. Cigarette smoking increases risk of Barrett's esophagus: an analysis of the Barrett's and Esophageal Adenocarcinoma Consortium. Gastroenterology. 2012;142(4):744-53.

9. Tran B, Lucas R, Kimlin M, Whiteman D, Neale R, Australian Cancer S. Association between ambient ultraviolet radiation and risk of esophageal cancer. Am J Gastroenterol. 2012;107(12):1803-13. 
10. Mulholland HG, Murray LJ, Anderson LA, Cantwell MM, group Fs. Vitamin D, calcium and dairy intake, and risk of oesophageal adenocarcinoma and its precursor conditions. Br J Nutr. 2011;106(5):732-41

11. Zgaga L, O'Sullivan F, Cantwell MM, Murray LJ, Thota PN, Coleman HG. Markers of Vitamin D Exposure and Esophageal Cancer Risk: A Systematic Review and Meta-analysis. Cancer Epidemiol Biomarkers Prev. 2016;25(6):877-86.

12. Smith GD, Ebrahim S. 'Mendelian randomization': can genetic epidemiology contribute to understanding environmental determinants of disease? International journal of epidemiology. 2003;32(1):1-22.

13. Lawlor DA, Harbord RM, Sterne JA, Timpson N, Davey Smith G. Mendelian randomization: using genes as instruments for making causal inferences in epidemiology. Stat Med. 2008;27(8):1133-63. 14. Greenland S. An introduction to instrumental variables for epidemiologists. International journal of epidemiology. 2000;29(4):722-9.

15. Burgess S, Butterworth A, Thompson SG. Mendelian randomization analysis with multiple genetic variants using summarized data. Genetic epidemiology. 2013;37(7):658-65.

16. Jiang X, O'Reilly PF, Aschard H, Hsu YH, Richards JB, Dupuis J, et al. Genome-wide association study in 79,366 European-ancestry individuals informs the genetic architecture of 25hydroxyvitamin D levels. Nature communications. 2018;9(1):260.

17. Gharahkhani P, Fitzgerald RC, Vaughan TL, Palles C, Gockel I, Tomlinson I, et al. Genome-wide association studies in oesophageal adenocarcinoma and Barrett's oesophagus: a large-scale meta-analysis. The Lancet Oncology. 2016;17(10):1363-73.

18. Levine DM, Ek WE, Zhang R, Liu X, Onstad L, Sather C, et al. A genome-wide association study identifies new susceptibility loci for esophageal adenocarcinoma and Barrett's esophagus. Nature genetics. 2013;45(12):1487-93. 
19. Schmermund A, Mohlenkamp S, Stang A, Gronemeyer D, Seibel R, Hirche H, et al. Assessment of clinically silent atherosclerotic disease and established and novel risk factors for predicting myocardial infarction and cardiac death in healthy middle-aged subjects: rationale and design of the Heinz Nixdorf RECALL Study. Risk Factors, Evaluation of Coronary Calcium and Lifestyle. Am Heart J. 2002;144(2):212-8

20. Palles C, Chegwidden L, Li X, Findlay JM, Farnham G, Castro Giner F, et al. Polymorphisms near TBX5 and GDF7 are associated with increased risk for Barrett's esophagus. Gastroenterology. 2015;148(2):367-78.

21. Didelez V MS, Sheehan NA. Assumptions of IV Methods for Observational Epidemiology. Stat Sci. 2010;25:22-40.

22. Thomas DC, Lawlor DA, Thompson JR. Re: Estimation of bias in nongenetic observational studies using "Mendelian triangulation" by Bautista et al. Ann Epidemiol. 2007;17(7):511-3.

23. Burgess S, Bowden J, Fall T, Ingelsson E, Thompson SG. Sensitivity Analyses for Robust Causal Inference from Mendelian Randomization Analyses with Multiple Genetic Variants. Epidemiology. 2017;28(1):30-42.

24. Afzal S, Brondum-Jacobsen P, Bojesen SE, Nordestgaard BG. Genetically low vitamin D concentrations and increased mortality: Mendelian randomisation analysis in three large cohorts. BMJ. 2014;349:g6330.

25. Pierce BL, Burgess S. Efficient design for Mendelian randomization studies: subsample and 2sample instrumental variable estimators. American journal of epidemiology. 2013;178(7):1177-84.

26. Burgess S, Small DS, Thompson SG. A review of instrumental variable estimators for Mendelian randomization. Stat Methods Med Res. 2017;26(5):2333-55.

27. Sheehan NA, Didelez V, Burton PR, Tobin MD. Mendelian randomisation and causal inference in observational epidemiology. PLoS Med. 2008;5(8):e177. 
28. Bowden J, Davey Smith G, Burgess S. Mendelian randomization with invalid instruments: effect estimation and bias detection through Egger regression. International journal of epidemiology. 2015;44(2):512-25.

29. Bowden J, Davey Smith G, Haycock PC, Burgess S. Consistent Estimation in Mendelian Randomization with Some Invalid Instruments Using a Weighted Median Estimator. Genetic epidemiology. 2016;40(4):304-14.

30. Staley JR, Blackshaw J, Kamat MA, Ellis S, Surendran P, Sun BB, et al. PhenoScanner: a database of human genotype-phenotype associations. Bioinformatics. 2016;32(20):3207-9.

31. Dimitrakopoulou VI, Tsilidis KK, Haycock PC, Dimou NL, Al-Dabhani K, Martin RM, et al. Circulating vitamin D concentration and risk of seven cancers: Mendelian randomisation study. BMJ. 2017;359:j4761.

32. Dudding T, Johansson M, Thomas SJ, Brennan P, Martin RM, Timpson NJ. Assessing the causal association between 25-hydroxyvitamin D and the risk of oral and oropharyngeal cancer using Mendelian randomization. Int J Cancer. 2018.

33. Ong JS, Cuellar-Partida G, Lu Y, Australian Ovarian Cancer S, Fasching PA, Hein A, et al. Association of vitamin D levels and risk of ovarian cancer: a Mendelian randomization study. International journal of epidemiology. 2016;45(5):1619-30.

34. Abnet CC, Chen Y, Chow WH, Gao YT, Helzlsouer KJ, Le Marchand L, et al. Circulating 25hydroxyvitamin D and risk of esophageal and gastric cancer: Cohort Consortium Vitamin D Pooling Project of Rarer Cancers. American journal of epidemiology. 2010;172(1):94-106.

35. Cummings LC, Thota PN, Willis JE, Chen Y, Cooper GS, Furey N, et al. A nonrandomized trial of vitamin D supplementation for Barrett's esophagus. PloS one. 2017;12(9):e0184928.

36. Thota PN, Kistangari G, Singh P, Cummings L, Hajifathalian K, Lopez R, et al. Serum 25Hydroxyvitamin D Levels and the Risk of Dysplasia and Esophageal Adenocarcinoma in Patients with Barrett's Esophagus. Dig Dis Sci. 2016;61(1):247-54. 


\section{Acknowledgements}

The following UK hospitals participated in sample collection through the Stomach and Esophageal Cancer Study (SOCS) collaboration network: Addenbrooke's Hospital, University College London, Bedford Hospital, Hinchingbrooke Hospital, Peterborough City Hospital, West Suffolk Hospital, Norfolk and Norwich University Hospital, Churchill Hospital, John Radcliffe Hospital, Velindre Hospital, St Bartholomew's Hospital, Queen's Hospital Burton, Queen Elisabeth Hospital, Diana Princess of Wales, Scunthorpe General Hospital, Royal Devon \& Exeter Hospital, New Cross Hospital, Belfast City Hospital, Good Hope Hospital, Heartlands Hospital, South Tyneside District General Hospital, Cumberland Infirmary, West Cumberland Hospital, Withybush General Hospital, Stoke Mandeville Hospital, Wycombe General Hospital, Wexham Park Hospital, Southend Hospital, Guy's Hospital, Southampton General Hospital, Bronglais General Hospital, Aberdeen Royal Infirmary, Manor Hospital, Clatterbridge Centre for Oncology, Lincoln County Hospital, Pilgrim Hospital, Grantham \& District Hospital, St Mary's Hospital London, Croydon University Hospital, Whipps Cross University Hospital, Wansbeck General Hospital, Hillingdon Hospital, Milton Keynes General Hospital, Royal Gwent Hospital, Tameside General Hospital, Castle Hill Hospital, St Richard's Hospital, Ipswich Hospital, St Helens Hospital, Whiston Hospital, Countess of Chester Hospital, St Mary's Hospital IOW, Queen Alexandra Hospital, Glan Clwyd Hospital, Wrexham Maelor Hospital, Darent Valley Hospital, Royal Derby Hospital, Derbyshire Royal Infirmary, Scarborough General Hospital, Kettering General Hospital, Kidderminster General Hospital, Royal Lancaster Infirmary, Furness General Hospital, Westmorland General Hospital, James Cook University Hospital, Friarage Hospital, Stepping Hill Hospital, St George's Hospital London, Doncaster Royal Infirmary, Maidstone Hospital, Tunbridge Hospital, Prince Charles Hospital, Hartlepool Hospital, University Hospital of North Tees, Ysbyty Gwynedd, St. Jame's University Hospital, Leeds General Infirmary, North Hampshire Hospital, Royal Preston Hospital, Chorley and District General, Airedale General Hospital, Huddersfield Royal Infirmary, Calderdale Royal Hospital, Torbay District General Hospital, Leighton Hospital, Royal Albert Edward Infirmary, Royal Surrey 
County Hospital, Bradford Royal Infirmary, Burnley General Hospital, Royal Blackburn Hospital, Royal Sussex County Hospital, Freeman Hospital, Royal Victoria Infirmary, Victoria Hospital Blackpool, Weston Park Hospital, Royal Hampshire County Hospital, Conquest Hospital, Royal Bournemouth General Hospital, Mount Vernon Hospital, Lister Hospital, William Harvey Hospital, Kent and Canterbury Hospital, Great Western Hospital, Dumfries and Galloway Royal Infirmary, Poole General Hospital, St Hellier Hospital, North Devon District Hospital, Salisbury District Hospital, Weston General Hospital, University Hospital Coventry, Warwick Hospital, George Eliot Hospital, Alexandra Hospital, Nottingham University Hospital, Royal Chesterfield Hospital, Yeovil District Hospital, Darlington Memorial Hospital, University Hospital of North Durham, Bishop Auckland General Hospital, Musgrove Park Hospital, Rochdale Infirmary, North Manchester General, Altnagelvin Area Hospital, Dorset County Hospital, James Paget Hospital, Derriford Hospital, Newham General Hospital, Ealing Hospital, Pinderfields General Hospital, Clayton Hospital, Dewsbury \& District Hospital, Pontefract General Infirmary, Worthing Hospital, Macclesfield Hospital, University Hospital of North Staffordshire, Salford Royal Hospital, Royal Shrewsbury Hospital, Manchester Royal Infirmary. 
Table 1. Characteristics of genetic variants associated with 25(OH)D concentration in published genome-wide association studies (ref.16)

\begin{tabular}{lllcclll}
\hline SNP & Chr & Gene & $\begin{array}{c}\text { Effect/ } \\
\text { Reference Allele }\end{array}$ & $\begin{array}{c}\text { Effect Allele } \\
\text { Frequency }\end{array}$ & $\begin{array}{l}\boldsymbol{\beta} \\
\text { estimate* }\end{array}$ & s.e. & P value \\
\hline rs10741657 & 11 & CYP2Rl & $\mathrm{A} / \mathrm{G}$ & 0.40 & 0.031 & 0.0022 & $2.1 \times 10^{-46}$ \\
rs10745742 & 12 & AMDHD1 & $\mathrm{T} / \mathrm{C}$ & 0.40 & 0.017 & 0.0022 & $1.9 \times 10^{-14}$ \\
rs12785878 & 11 & DHCR7 & $\mathrm{T} / \mathrm{G}$ & 0.75 & 0.036 & 0.0022 & $3.8 \times 10^{-62}$ \\
rs17216707 & 20 & CYP24A1 & $\mathrm{T} / \mathrm{C}$ & 0.79 & 0.026 & 0.0027 & $8.1 \times 10^{-23}$ \\
rs3755967 & 4 & GC & $\mathrm{C} / \mathrm{T}$ & 0.62 & 0.089 & 0.0023 & $4.7 \times 10^{-343}$ \\
rs8018720 & 14 & SEC23A & $\mathrm{G} / \mathrm{C}$ & 0.18 & 0.017 & 0.0029 & $4.7 \times 10^{-9}$ \\
\hline
\end{tabular}

$* 25(\mathrm{OH}) \mathrm{D}$ level was reported SD increases in $\mathrm{nmol} / \mathrm{L}$ in natural-log scale per copy of the effect alleles. 
Table 2. Characteristics of the studies included for the gene-25(OH)D and gene-BE/EAC associations

\begin{tabular}{|c|c|c|}
\hline Data Source & $n$ studies & Sample size \\
\hline \multicolumn{3}{|l|}{ Gene-Vitamin D association } \\
\hline SUNLIGHT Consortium (ref.16) & 31 & 79,366 \\
\hline \multicolumn{3}{|l|}{ Gene-BE/EAC associations } \\
\hline BEACON (ref. 17, 18) & 15 & $\begin{array}{l}2,406 \mathrm{BE}, 1,508 \mathrm{EAC}, 6,718 \\
\text { controls }\end{array}$ \\
\hline - United States & 9 & 1,718 BE, 668 EAC, 1,021 controls \\
\hline - United Kingdom & 1 & $167 \mathrm{BE}, 102 \mathrm{EAC}$ \\
\hline - Sweden & 1 & $63 \mathrm{EAC}, 116$ controls \\
\hline - Canada & 1 & 246 EAC, 256 controls \\
\hline - Australia & 2 & $324 \mathrm{BE}, 236 \mathrm{EAC}, 567$ controls \\
\hline - Ireland & 1 & $197 \mathrm{BE}, 193 \mathrm{EAC}, 217$ controls \\
\hline - dbGap & 1 & 4,541 controls \\
\hline Bonn (Germany) (ref. 17, 19) & 1 & $\begin{array}{l}1,037 \mathrm{BE}, 1,609 \mathrm{EAC}, 3,537 \\
\text { controls }\end{array}$ \\
\hline Cambridge (UK) (ref.17) & 1 & $873 \mathrm{BE}, 995 \mathrm{EAC}, 3,408$ controls \\
\hline Oxford (UK) (ref.17, 20) & 7 & $1,851 \mathrm{BE}, 3,496$ controls \\
\hline
\end{tabular}


Table 3. Characteristics of genetic variants associated with Barrett's esophagus and esophageal adenocarcinoma

\begin{tabular}{llllcccc}
\hline \multirow{2}{*}{ SNP } & \multirow{2}{*}{ Chr } & \multirow{2}{*}{ Gene } & Effect/ & \multicolumn{2}{c}{ BE } & \multicolumn{2}{c}{ EAC } \\
\cline { 6 - 8 } & & & Reference Allele & OR (95\% CI) & P & OR (95\% CI) & P \\
\hline rs10741657 & 11 & CYP2R1 & $\mathrm{A} / \mathrm{G}$ & $1.01(0.97-1.06)$ & 0.577 & $0.98(0.93-1.04)$ & 0.465 \\
rs10745742 & 12 & AMDHD1 & $\mathrm{T} / \mathrm{C}$ & $0.97(0.92-1.01)$ & 0.122 & $1.01(0.95-1.06)$ & 0.811 \\
rs12785878 & 11 & DHCR7 & $\mathrm{T} / \mathrm{G}$ & $1.07(1.02-1.13)$ & 0.008 & $0.99(0.93-1.05)$ & 0.785 \\
rs17216707 & 20 & CYP24A1 & $\mathrm{T} / \mathrm{C}$ & $0.98(0.92-1.04)$ & 0.495 & $1.00(0.93-1.08)$ & 0.920 \\
rs3755967 & 4 & GC & $\mathrm{C} / \mathrm{T}$ & $1.00(0.96-1.05)$ & 0.844 & $0.96(0.91-1.02)$ & 0.213 \\
rs8018720 & 14 & SEC23A & $\mathrm{G} / \mathrm{C}$ & $1.01(0.95-1.06)$ & 0.841 & $0.99(0.92-1.06)$ & 0.778 \\
\hline
\end{tabular}


Supplementary Table 1. Mendelian randomization estimates using MR-Egger and weighted median approach

\begin{tabular}{llcl}
\hline \multirow{2}{*}{ Disease } & \multicolumn{2}{c}{ MR-Egger } & Weighted median \\
\cline { 2 - 4 } & ${\text { Intercept } \boldsymbol{P} \text { value }^{\mathrm{a}}}$ & Slope OR (95\% CI) & \\
\hline BE & 0.793 & $1.32(0.59-2.98)$ & $1.12(0.67-1.88)$ \\
EAC & 0.749 & $0.60(0.22-1.61)$ & $0.66(0.36-1.23)$ \\
\hline
\end{tabular}

${ }^{\text {a }}$ The $P$-value of the intercept is as a valid test of directional pleiotropy.

${ }^{b}$ The slope of the MR-Egger regression is the pleiotropy-adjusted causal effect estimate.

${ }^{c}$ The OR represent increase/decrease of risk per $20 \mathrm{nmol} / \mathrm{L}$ increase of $25(\mathrm{OH}) \mathrm{D}$. 
Supplementary Table 2. Associations of the 25(OH)D-associated SNPs with other phenotypes

\begin{tabular}{|c|c|c|c|c|c|c|}
\hline SNP & Chr & Gene & Phenotype & Beta & $\boldsymbol{P}$ & PMID \\
\hline \multirow[t]{4}{*}{ rs10741657 } & 11 & $C Y P 2 R 1$ & Hip circumference & 0.015 & $1.10 \mathrm{E}-03$ & 25673412 \\
\hline & & & Height & 0.009 & $2.00 \mathrm{E}-03$ & 25282103 \\
\hline & & & Conscientiousness & 0.232 & $2.22 \mathrm{E}-03$ & 21173776 \\
\hline & & & $\log (\mathrm{eGFR}$ creatinine in non diabetics) & -0.003 & $2.60 \mathrm{E}-03$ & 26831199 \\
\hline \multirow[t]{6}{*}{ rs10745742 } & 12 & $A M D H D 1$ & Subject well being & -0.011 & $1.28 \mathrm{E}-03$ & 27089181 \\
\hline & & & 2 hour fasting glucose & -0.055 & $3.58 \mathrm{E}-03$ & 20081857 \\
\hline & & & Birthweight & 0.022 & $9.10 \mathrm{E}-03$ & 23202124 \\
\hline & & & Visual refractive error & 0.089 & $9.22 \mathrm{E}-03$ & 23474815 \\
\hline & & & Extraversion & -0.014 & $1.24 \mathrm{E}-02$ & 26362575 \\
\hline & & & Total cholesterol & NA & $1.66 \mathrm{E}-02$ & 20339536 \\
\hline \multirow[t]{4}{*}{ rs12785878 } & 11 & DHCR7 & $\begin{array}{l}\text { Attention deficit hyperactivity } \\
\text { disorder }\end{array}$ & NA & $4.03 \mathrm{E}-05$ & 20732625 \\
\hline & & & Waist hip ratio in males $\leq 50$ years & 0.025 & $9.00 \mathrm{E}-03$ & 26426971 \\
\hline & & & Total cholesterol & -0.014 & $1.41 \mathrm{E}-02$ & 24097068 \\
\hline & & & $\log (\mathrm{eGFR}$ creatinine $)$ & 0.002 & $1.70 \mathrm{E}-02$ & 26831199 \\
\hline \multirow[t]{5}{*}{ rs17216707 } & 20 & $C Y P 24 A 1$ & $\log (\mathrm{eGFR}$ creatinine $)$ & 0.008 & $6.00 \mathrm{E}-13$ & 26831199 \\
\hline & & & Serum creatinine & NA & $8.90 \mathrm{E}-06$ & 20383146 \\
\hline & & & Femoral neck bone mineral density & NA & $8.26 \mathrm{E}-04$ & 22504420 \\
\hline & & & Cystatin $\mathrm{C}$ in serum & NA & $7.50 \mathrm{E}-03$ & 20383146 \\
\hline & & & Type II diabetes & 0.049 & $1.10 \mathrm{E}-02$ & 24509480 \\
\hline \multirow[t]{6}{*}{ rs3755967 } & 4 & $G C$ & Height & -0.009 & 4.90E-03 & 25282103 \\
\hline & & & $\log$ (Proinsulin) & -0.021 & $6.14 \mathrm{E}-03$ & 21873549 \\
\hline & & & Alzheimers disease & 0.036 & $3.35 \mathrm{E}-02$ & 24162737 \\
\hline & & & Waist hip ratio in males $\leq 50$ years & -0.017 & $3.60 \mathrm{E}-02$ & 26426971 \\
\hline & & & Chronic kidney disease & NA & $3.60 \mathrm{E}-02$ & 20383146 \\
\hline & & & Years of educational attainment & 0.009 & $3.63 \mathrm{E}-02$ & 23722424 \\
\hline \multirow[t]{8}{*}{ rs8018720 } & 14 & $S E C 23 A$ & Rheumatoid arthritis & 0.083 & $8.43 \mathrm{E}-03$ & 20453842 \\
\hline & & & Hip circumference in males & 0.02 & $2.10 \mathrm{E}-02$ & 25673412 \\
\hline & & & Waist hip ratio tails & -0.1 & $3.20 \mathrm{E}-02$ & 23563607 \\
\hline & & & Tardive dyskinesia & NA & 3.63E-02 & 19875103 \\
\hline & & & Gout & -0.087 & 4.22E-02 & 23263486 \\
\hline & & & Body fat percentage & -0.014 & $4.40 \mathrm{E}-02$ & 26833246 \\
\hline & & & Simpson Angus Scale & NA & $4.47 \mathrm{E}-02$ & 19875103 \\
\hline & & & $\log (\mathrm{DI})$ & 0.055 & 4.62E-02 & 24699409 \\
\hline
\end{tabular}

